\title{
Prolonged otorrhea and mastoiditis caused by Mycobacterium abscessus
}

\author{
Pelkonen, Tuula
}

2011

Pelkonen , T , Aarnisalo , A, Markkola , A , Eskola , J , Saxen , H \& Salo , E 2011 , '

Prolonged otorrhea and mastoiditis caused by Mycobacterium abscessus ' , International

Journal of Pediatric Otorhinolarynology. Extra , vol. 6 , no. 4 , pp. 388-391 . https://doi.org/10.1016/j.pedex.2011.05.

http://hdl.handle.net/10138/299301

https://doi.org/10.1016/j.pedex.2011.05.002

publishedVersion

Downloaded from Helda, University of Helsinki institutional repository.

This is an electronic reprint of the original article.

This reprint may differ from the original in pagination and typographic detail.

Please cite the original version. 
Case report

\title{
Prolonged otorrhea and mastoiditis caused by Mycobacterium abscessus
}

\author{
Tuula Pelkonen $^{\mathrm{a}, \mathrm{b}, *}$, Antti Aarnisalo ${ }^{\mathrm{c}}$, Antti Markkola ${ }^{\mathrm{d}}$, Jussi Eskola ${ }^{\mathrm{e}}$, Harri Saxen ${ }^{\mathrm{a}}$, Eeva Salo ${ }^{\mathrm{a}}$ \\ ${ }^{a}$ Helsinki University Central Hospital, Hospital for Children and Adolescents, Helsinki, Finland \\ ${ }^{\mathrm{b}}$ University of Helsinki, Helsinki, Finland \\ ${ }^{\mathrm{c}}$ Helsinki University Central Hospital, Department of Otorhinolaryngology, Helsinki, Finland \\ ${ }^{\mathrm{d}}$ Helsinki University Central Hospital, Department of Radiology, Helsinki, Finland \\ ${ }^{\mathrm{e}}$ Helsinki University Central Hospital Laboratory (HUSLAB), Mycobacteriology Unit, Helsinki, Finland
}

\section{A R T I C L E I N F O}

\section{Article history:}

Received 1 March 2011

Accepted 3 May 2011

Available online 28 May 2011

\section{Keywords:}

Nontuberculous mycobacteria

Mycobacterium abscessus

Mastoiditis

Child

\begin{abstract}
A B S T R A C T
Infections caused by nontuberculous mycobacteria (NTM) have been reported increasingly. Rarely, NTM also cause chronic ear infections. We describe Mycobacterium abscessus mastoiditis in an immunocompetent child, whose painless chronic otorrhea failed to settle with routine antimicrobial and local therapy. Polyps and granulation tissue were noted on the tympanic membrane. The diagnosis was made with staining on acid-fast bacilli and culture of mycobacteria in biopsy material. The successful treatment consisted of surgery, removal of foreign material (tympanostomy tube), and antimicrobials. Chronic otorrhea unresponsive to standard therapy can be caused by NTM and should be examined for the presence of acid-fast bacilli.
\end{abstract}

() 2011 Elsevier Ireland Ltd. All rights reserved.

\section{Introduction}

In recent years, infections caused by nontuberculous mycobacteria (NTM) have been reported increasingly. Whether this is due to increasing prevalence or improved diagnostics remains unclear [1]. In countries like Sweden where routine BCG vaccinations have been suspended, the numbers of infections caused by NTM have increased [2]. More than 120 species of NTM have been recognized; many of them are ubiquitous and found in soil, food, water, and animals [1,3].

In children, the most common infection caused by NTM is cervical lymphadenitis [4]; others involve skin, bones, lungs, central nervous system, or eye. NTM enter the body through skin abrasions, surgical trauma, oropharyngeal mucosa, and gastrointestinal or respiratory tract. Most infections localize at the portal of entry, or spread to the local lymph nodes. Impaired cell-mediated immunity predisposes the host to disseminated infection $[1,3]$. Traditional antituberculous medicines are usually not effective against NTM, and these infections are often difficult to treat [5].

Rarely, NTM can also cause chronic ear infections and mastoiditis [6-10]. Mycobacterium abscessus belongs to the rapidly

\footnotetext{
* Corresponding author at: Helsinki University Central Hospital, Hospital for Children and Adolescents, PL 281, 00029, HUS, Helsinki, Finland.

Tel.: +358 504140758 .

E-mail addresses: tuula.i.pelkonen@hus.fi, tuulapelkonen@hotmail.com (T. Pelkonen).
}

growing mycobacteria and is considered to be the most dangerous, pathogenic, and therapy-resistant of the NTMs [5].

We describe a child with mastoiditis caused by M. abscessus.

\section{Case report}

A 5-year old boy had a history of recurrent otitis media and therefore in January 2009 ventilation tubes were installed in both tympanic membranes. In and between March and May, the parents noted otorrhea from the left ear 4-5 times. In June, granulation tissue was noted on the left tympanic membrane (TM) on otorhinolaryngologic examination. After a benign respiratory infection in October, persistent otorrhea was recorded and treated with oral amoxycillin, saline irrigations and chloramphenicol ear drops. Bacterial culture of the secretion showed normal bacterial flora. The boy was referred to our hospital November 25th because of persisting otorrhoea. Before that the boy had had fever up to $38.5^{\circ} \mathrm{C}$ for 3 weeks, headache, and redness of the skin behind left ear for 2 days.

At presentation left-sided auricular proptosis (Fig. 1), postauricular erythema and tenderness were observed. C-reactive protein (CRP) was $24 \mathrm{mg} / \mathrm{L}$. Otoscopy showed edematous external auditory canal filled with purulent secretion, and otomicroscopy revealed granulation on auditory canal and polyps on TM. The boy was treated with intravenous cefuroxime and prednisolon, and discharged two days later with oral cefalexin and prednisolon; chloramphenicol ear drops were continued.

The patient returned to the hospital because of continuing fever two days after discharge. CRP had risen to $43 \mathrm{mg} / \mathrm{L}$. Computed 




Fig. 1. Left-sided auricular proptosis caused by mastoiditis.

tomography (CT) showed opacification of left middle ear and mastoid ear cells in combination with destructive changes of mastoid septations and external mastoid cortex (Fig. 2). Magnetic resonance imaging (MRI) demonstrated more distinctively the inflammatory tissue changes with a subperiosteal abscess and a reactive inflammatory thickening of left temporal lobe dura (Fig. 3).

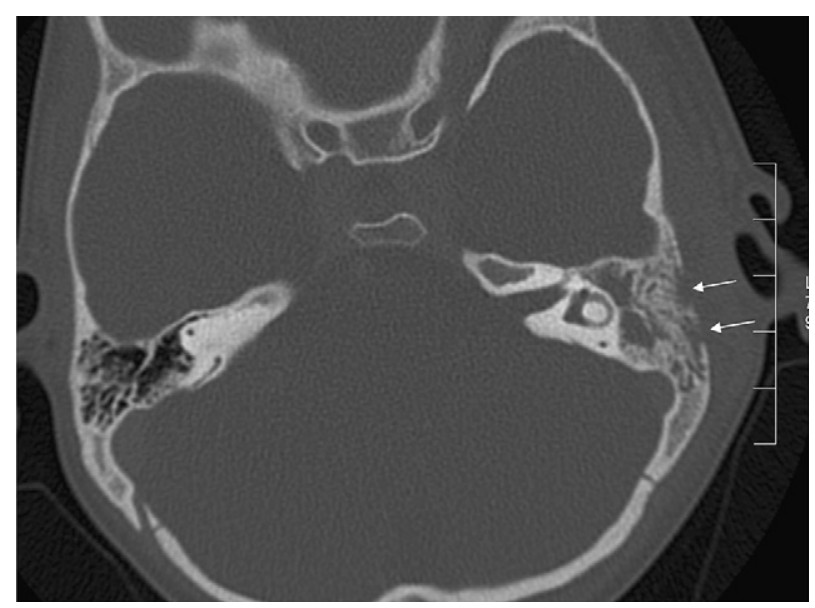

Fig. 2. Axial bone $\mathrm{CT}$ of left ear erosive otomastoiditis shows opacification of middle ear and mastoid ear cells. Mastoid septations and external mastoid cortex are partially destroyed (arrows).

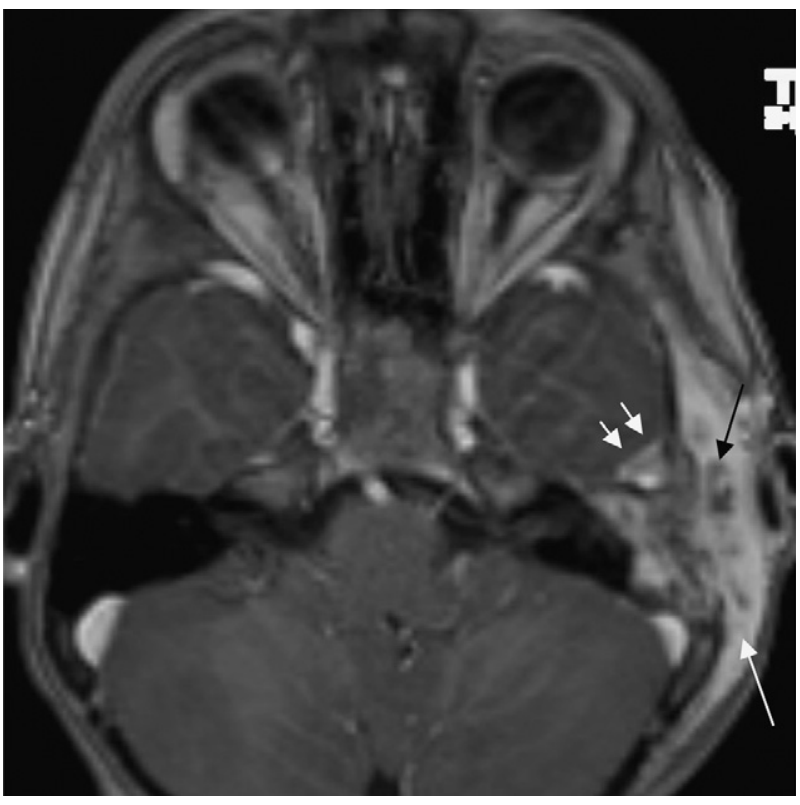

Fig. 3. Axial T1 contrast enhanced MR image of left ear erosive otomastoiditis shows diffuse enhancement of subcutaneous inflammatory tissue (white arrow). Nonenhancing subperiosteal abscess (black arrow) is seen within inflammatory tissue lateral to the opacified mastoid air cells. Notice also the thickened, enhancing dura of left temporal lobe (two arrows).

Tympanomastoidectomy performed three days later revealed edema of the subcutaneous tissue, purulent secretion in the external auditory canal, edema and granulation on TM, and mucosal edema and purulent secretion in the mastoid process. A new ventilation tube of titanum was installed. Mucosal biopsy taken from the mastoid cavity showed granulation tissue. The antibiotic treatment consisted of intravenous ceftriaxone and metronidazole, and ciprofloxacin + hydrocortison ear drops. In a follow-up MRI inflammatory changes had diminished. Otomicroscopy five days after the surgery showed edema of the external auditory canal; and when the stitches behind the ear were removed, the wound opened, and revealed granulating tissue with serous secretion. The boy was discharged the next day with local wound treatment, daily ceftriaxone infusions, and ciprofloxacin + hydrocortison ear drops. Results of bacterial staining and culture taken from the mastoid cavity revealed acid-fast bacilli, and the boy was called to the pediatric hospital the following day.

A careful interview disclosed a grandmother who had suffered from pulmonary tuberculosis, but passed away before the boy was born. The boy was vaccinated with BCG, and had a scar of vaccination. He was a very eager swimmer, and used to swim in swimming pools and lakes. His erythrocyte sedimentation rate (ESR) was $38 \mathrm{~mm} / \mathrm{h}$, tuberculin skin test with Mantoux technique $6 \mathrm{~mm}$, and chest X-ray normal. In vitro lymphocyte stimulation test with Mycobacterium tuberculosis antigens (TBSpot ${ }^{\mathrm{TM}}$ ) was negative for tuberculosis. Treatment with oral clarithromycin and rifabutin was started.

At follow-up visit two weeks later the external auditory canal was moist, edema had diminished, and the retroauricular wound was granulating. The boy had had fever $38.7^{\circ} \mathrm{C}$. ESR had lowered to $29 \mathrm{~mm} / \mathrm{h}$, and CPR to normal. Final result of the bacterial culture was available: M. abscessus which was sensitive to clarithromycin and amikacin; resistant to doxicyclin, cefoxitin and ciprofloxacin. Rifabutin (not tested) was discontinued.

A week later the wound appeared wider and more inflamed (Fig. 4); the external auditory canal continued macerated and with 


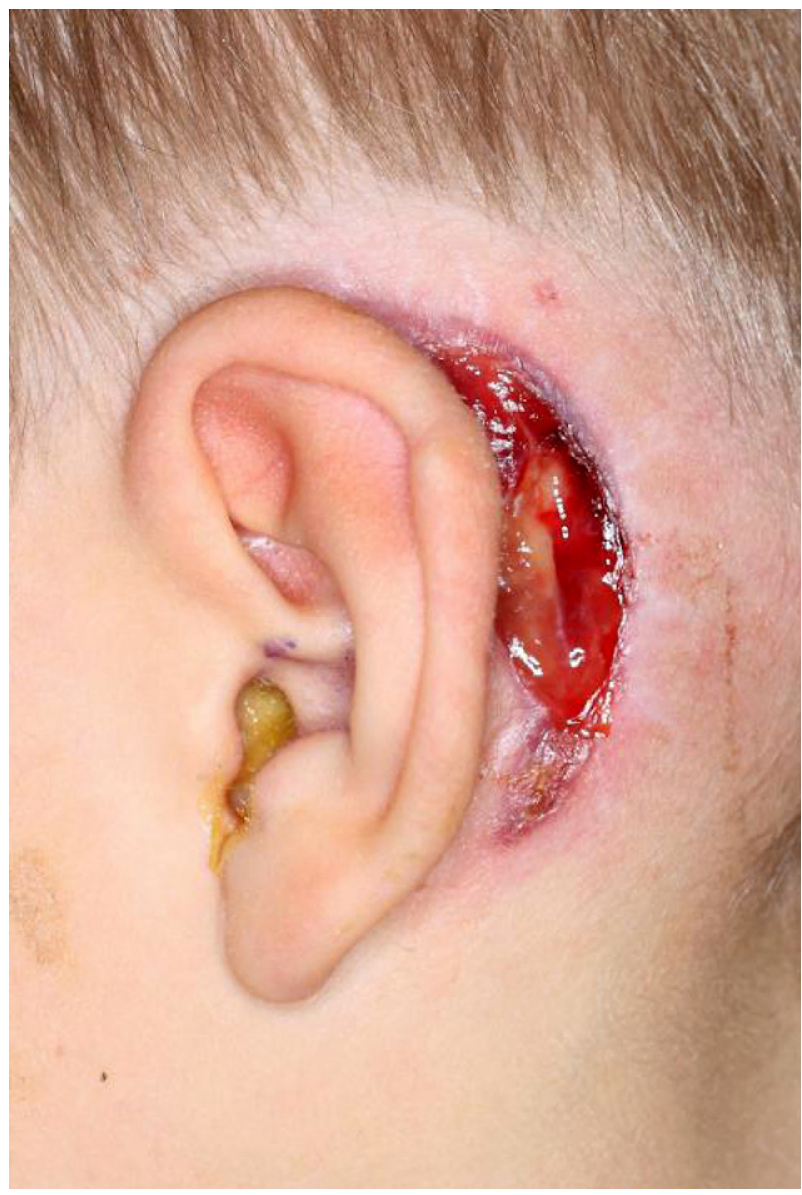

Fig. 4. Unhealed and discharging wound with granulation tissue after mastoidectomy.

edema; ESR was $13 \mathrm{~mm} / \mathrm{h}$, and CRP normal. The tympanostomy tube was removed and clarithromycin continued. In addition amikacin once daily intravenously was started.

Three weeks later the boy's temperature was $37.1^{\circ} \mathrm{C}$, and he was gaining weight. The wound was dry and had started to heal; TM was visible and normal. ESR and CRP had normalized. Amikacin treatment was discontinued, and clarithromycin continued. At the next follow-up visit in February, four weeks later, the boy had not had fever for 10 days, the wound had half closed, and otoscopy revealed normal auditory canal and TM. Clarithromycin treatment was continued for six months, until June, when the wound had healed and the boy had been without symptoms for four months. In April the hearing thresholds of the boy's right and left ear were 5 and $20 \mathrm{dBs}$, respectively. At follow-up visit in August the boy did not have any complaints or abnormal findings. All analyses of possible immune system disorders (immunoglobulins, complement activity, interleukin-12 and gamma-interferon stimulation tests) were normal.

\section{Discussion}

NTM are found everywhere in our environment: soil and dust, water, domestic and wild animals, dairy products and eggs. Human-to-human transmission has not been reported [8]. Our patient was a frequent swimmer. The most probable vehicle of the pathogen was contaminated water and the most likely route of entry through the tympanostomy tubes. Other reported possible routes of infection in mastoiditis include the external ear, from pharynx through eustachian tube, hematogenous route or through contaminated instruments.

Immunodeficiency may predispose the host to NTM infections. Mutations in the IL-12 and IFN- $\gamma$ receptors have been found in patients with disseminated and relapsing NTM infections [11]. However, otitis or mastoiditis has occurred mostly in immunocompetent children. Our patient showed no abnormal immunological tests.

Our patient showed typical signs of mastoiditis caused by NTM. The general condition of the child was good, and unilateral painless chronic otorrhea failed to settle with routine antimicrobial and local therapy. There were both polyps and granulation tissue in the external ear canal and on the tympanic membrane [10]. CT and MRI demonstrated destructive otomastoiditis: acute infection of middle ear and mastoid air cells with destructive changes of mastoid septations and external temporal bone cortex and a subperiosteal abscess. The unhealed and discharging wound with granulation tissue after the surgery is typical for NTM infection [10]. Differential diagnosis should include infection caused by $M$. tuberculosis.

The diagnosis is made with staining on acid-fast bacilli and culture of mycobacteria in tissue specimens or discharge samples [12]. Histology of the biopsy material shows granulomatous changes. In NTM infections tuberculin skin test can react moderately, but the induration is rarely more than $15 \mathrm{~mm}$ [3]. Interferon gamma release assay (IGRA) with $M$. tuberculosis specific antigens is usually negative in NTM infections.

Spontaneous recovery of NTM ear infection is rare. Any foreign material should be removed (tympanostomy tubes) and affected tissue surgically removed [7,12]. Antibiotic therapy should continue at least 2-3 months after disappearance of symptoms [8]. Clarithromycin and amikacin are the recommended drugs $[5,12]$. Some patients have been treated with clarithromycin monotherapy, but combination therapy with at least one other agent (amikacin, cefoxitin, ciprofloxacin, rifampin, ethambutol, trimetoprim-sulfamethoxazole, doxycycline, or meropenem) is usually recommended $[3,9]$.

In summary, chronic painless otorrhea in children unresponsive to standard therapy can be caused by NTM and should be examined for the presence of acid-fast bacilli.

\section{Conflict of interest}

None of the authors has conflicts of interest.

\section{References}

[1] C. Piersimoni, C. Scarparo, Extrapulmonary infections associated with nontuberculous mycobacteria in immunocompetent persons, Emerg. Infect. Dis. 15 (2009) 1351.

[2] V. Romanus, H.O. Hallander, P. Wahlen, A.M. Olinder-Nielsen, P.H. Magnusson, I. Juhlin, Atypical mycobacteria in extrapulmonary disease among children. Incidence in Sweden from 1969 to 1990, related to changing BCG-vaccination coverage, Tuberc. Lung Dis. 76 (1995) 300-310.

[3] American Academy of Pediatrics, Diseases caused by nontuberculous mycobacteria, in: L.K. Pickering, C.J. Baker, D.W. Kimberlin, S.S. Long (Eds.), Red Book: 2009 Report of the Committee on Infectious Diseases, 28th ed., American Academy of Pediatrics, Elk Grove Village, IL, 2009, pp. 701-708.

[4] M.K. Timmerman, A.D. Morley, J. Buwalda, Treatment of non-tuberculous mycobacterial cervicofacial lymphadenitis in children: critical appraisal of the literature, Clin. Otolaryngol. 33 (2008) 546-552.

[5] B. Petrini, Mycobacterium abscessus: an emerging rapid-growing potential pathogen, APMIS 114 (2006) 319-328.

[6] A. van Aarem, H.L. Muytjens, M.M. Smits, C.W. Cremers, Recurrent therapy resistant mastoiditis by Mycobacterium cheilonae abscessus, a nontuberculous mycobacterium, Int. J. Pediatr. Otorhinolaryngol. 43 (1998) 61-72.

[7] J.J. Linmans, R.J. Stokroos, C.F. Linssen, Mycobacterium abscessus, an uncommon cause of chronic otitis media: a case report and literature review, Arch. Otolaryngol. Head Neck Surg. 134 (2008) 1004-1006.

[8] B. Muller, J. Kemper, N.G. Hartwig, E.A. Mooi-Kokenberg, R. van Altena, F.G. Versteegh, Mycobacterium avium intracellulare otomastoiditis: case report and literature review, Eur. J. Clin. Microbiol. Infect. Dis. 25 (2006) 723-727. 
[9] J. van Ingen, F. Looijmans, P. Mirck, R. Dekhuijzen, M. Boeree, D. van Soolingen, Otomastoiditis caused by Mycobacterium abscessus, the Netherlands, Emerg. Infect. Dis. 16 (2010) 166-168.

[10] D. Flint, M. Mahadevan, R. Gunn, S. Brown, Nontuberculous mycobacterial otomastoiditis in children: four cases and a literature review, Int. J. Pediatr. Otorhinolaryngol. 51 (1999) 121-127.
[11] R. de Jong, F. Altare, I.A. Haagen, et al., Severe mycobacterial and salmonella infections in interleukin-12 receptor-deficient patients, Science 280 (1998) 1435-1438.

[12] D.E. Griffith, T. Aksamit, B.A. Brown-Elliott, et al., An official ATS/IDSA statement: diagnosis, treatment, and prevention of nontuberculous mycobacterial diseases, Am. J. Respir. Crit. Care Med. 175 (2007) 367-416. 